\title{
Systematic reviews of apical prolapse surgery: are we being misled down a dangerous path?
}

\author{
Michael Moen ${ }^{1}$ - John Gebhart ${ }^{2}$ - Karl Tamussino ${ }^{3}$
}

Received: 14 April 2015 / Accepted: 24 April 2015 / Published online: 12 May 2015

(C) The International Urogynecological Association 2015

"I was told I had the best procedure for my condition. Is it true I could have had vaginal surgery without mesh?" This question was posed by the 43-year-old woman now facing the need for adhesiolysis and trachelectomy due to pelvic pain and cyclic bleeding two years after undergoing laparoscopic supracervical hysterectomy and mesh sacrocervicopexy for stage 3 uterine prolapse (Fig. 1).

The claim of superiority of sacrocolpopexy (SC) over other pelvic organ prolapse (POP) procedures was initiated by the results of three randomized controlled trials (RCTs) involving a total of 287 patients comparing abdominal SC (ASC) and sacrospinous ligament fixation (SSLF) published in 1996, 1998 and 2004 [1-3]. These studies reported superior anatomic outcomes with ASC when compared with SSLF, but also showed higher short-term morbidity with ASC. The claim of superiority of ASC over SSLF was further reinforced with the publication of the first Cochrane systematic review of surgery for POP published in 2004, which summarized the findings of the three previously mentioned studies stating "abdominal sacral colpopexy was better than vaginal sacrospinous colpopexy in terms of a lower rate of recurrent vault prolapse and less dyspareunia, but the trend towards a lower reoperation rate for prolapse following abdominal sacrocolpopexy was not statistically significant" [4]. Since these initial RCTs comparing ASC with SSLF, several systematic reviews have shown the same findings of superior ana-

Michael Moen

Michael.Moen-MD@Advocatehealth.com

1 Illinois Urogynecology, Ltd, 1875 Dempster St, Suite 665, Park Ridge, IL 60068, USA

2 Mayo Clinic, Rochester, MN, USA

3 Medical University of Graz, Graz, Austria tomic outcomes associated with ASC compared with SSLF [4-11]. These reviews reinforce the perception that there is a large body of evidence supporting ASC as the best option for treating POP.

Laparoscopic SC (LSC) and robot-assisted SC have been developed to reduce the short-term morbidity associated with ASC $[12,13]$. Many surgeons consider these less invasive approaches to SC (and variations such as sacrocervicopexy) equivalent to ASC in terms of anatomic outcome leading them to claim that LSC and robotic SC approaches are now the best options for treating POP. The most recent Cochrane review published in 2013 concludes "sacral colpopexy has superior outcomes to a variety of vaginal procedures including sacrospinous colpopexy, uterosacral colpopexy and transvaginal mesh" implying that all forms of SC are superior to all other surgical options for treating apical prolapse [9].

A close look at the current literature, however, reveals that there are actually very few level 1 studies from which to derive such a bold conclusion concerning SC compared with other options for treating POP. Only two studies comparing SC with other procedures have been added to the Cochrane review over the past decade and both of these are controversial. One is a trial comparing LSC with total vaginal mesh (TVM) that showed superiority of LSC compared to TVM [14]. The study is controversial because the reported success rate for TVM was significantly lower than the rate achieved by other authors and because of this low rate, LSC was reported as superior. The other is an abstract of a trial comparing ASC with uterosacral ligament suspension (USLS) that showed superiority of ASC, but this study is yet to be published as a full peer-reviewed article limiting the ability to assess the quality and potential confounding factors affecting the results [15]. It is the addition of these two controversial studies which resulted in the change of wording in the Cochrane Review summary from the more specific statement that "ASC is superior to 


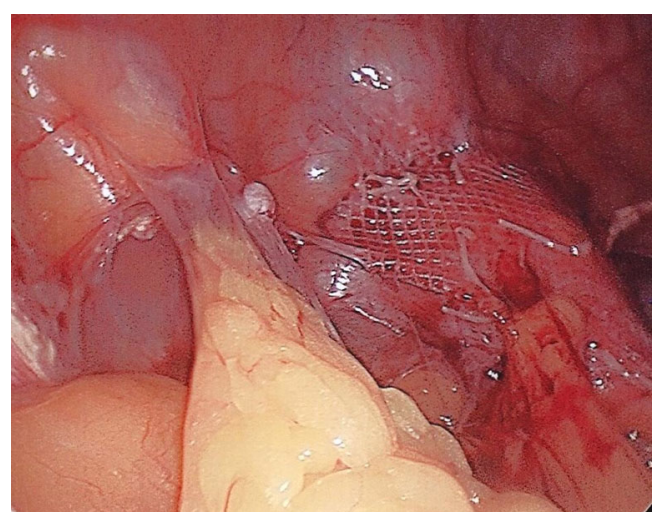

Fig. 1 Adhesions two years after laparoscopic sacrocervicopexy procedure

SSLF" to the more general statement that "SC is superior to multiple procedures including SSLF, USLS and TVM".

The strict constraints required for systematic reviews can result in a limited number of studies considered appropriate for analysis and inclusion in a review. To date, there are over twice as many publications of systematic reviews summarizing apical prolapse repair as there are RCTs used to derive the conclusions of these reviews [4-11]. The effect is the appearance of a large amount of high-quality data leading to the conclusion that $\mathrm{ASC} / \mathrm{SC}$ is superior to other options, but the reality is that these reviews repeat the same information from a small number of heterogeneous level 1 studies. This is an interesting form of duplicate/ multiple publication that creates an exaggerated perception of the literature supporting the superiority of SC. Coupled with older reports which portray high anatomic failure rates of native tissue repairs, it is unfortunate, but not surprising, that many urogynecologic surgeons have been influenced to shift their focus from traditional native tissue repairs to procedures utilizing mesh. A worrisome result of this shift is seen in training programs that put increasing focus on minimally invasive mesh techniques with a resultant decrease in experience with traditional vaginal repairs.

During the time these studies and systematic reviews have been published, significant changes have occurred in POP surgery research and reporting, most notably expansion of outcome measures beyond a primary focus on anatomic results as reported in older RCTs to more comprehensive patient-centered outcomes including symptoms, satisfaction, quality of life and patient safety factors, particularly risks of long-term complications. Studies with longer term follow-up indicate that anatomic outcomes for mesh and non-mesh apical procedures are similar [16-19]. In fact, the results from one of the most rigorous and respected RCTs concerning SC show that the rate of recurrent prolapse symptoms is similar to rates often quoted for traditional native tissue repairs and the mesh complication rate is similar to that seen with vaginal mesh procedures [19].
The addition of mesh, whether abdominally or vaginally, does not universally result in definitive cure of POP and the risk of mesh-related complications continues indefinitely. Systematic reviews of risks associated with the use of mesh in POP surgery indicate that mesh procedures ( $\mathrm{SC}$ and vaginal mesh) result in lower rates of reoperation for recurrent prolapse, but have higher reoperation rates for complications, resulting in a higher total reoperation rate with mesh than with native tissue repairs [20, 21]. Reoperation for gastrointestinal complications and mesh complications is typically more difficult than reoperation for recurrent prolapse alone and the level of difficulty of these procedures can be expected to be higher when the uterus and/or cervix is left in place, as was the case in the patient presented at the beginning of this article [22]. With this in mind, the decision to utilize permanent materials in primary POP procedures comes under greater scrutiny and the argument to judiciously use permanent materials in patients with recurrent prolapse is understandable. Clearly, further basic science research and more long-term comparative studies with appropriate outcome measures are needed to better determine the safe and effective use of permanent synthetic materials in POP surgery [23].

Although SC is reported as superior in systematic reviews, most clinicians utilize native tissue repairs, particularly vaginal hysterectomy with apical suspension and colporrhaphy as their primary approach to surgical management of POP, and reserve the use of mesh for patients with recurrence. The discrepancy between conclusions of current POP surgery research and worldwide practice patterns points out the need for better analysis and utilization of information from all useful sources including outcomes in everyday clinical practice. Collecting and analyzing the large body of data generated in practice through well-designed and monitored databases and registries such as the Austrian Registry, the British Society of Urogynaecology (BSUG) Database and the Pelvic Floor Disorders Registry (PFDR) in the United States, provides another source of evidence to utilize in answering important clinical questions. With over 200,000 POP surgery cases in the United States each year, capturing just $1 \%$ of these cases in a registry would provide over four times the total number of cases that were analyzed in the five RCTs referenced in the most recent Cochrane update. Furthermore, generalizability of results from RCTs can only be determined by comparing the results from these RCTs with actual outcomes in practice. Without this essential step, results from RCTs and systematic reviews risk being irrelevant to clinical practice. Certainly for the individual surgeon, knowledge of actual outcomes in practice is essential in guiding care and counseling patients.

In conclusion, several systematic reviews report $\mathrm{SC}$ as a superior procedure for apical repair, but the actual amount of level 1 data from which to make the claim is small and based on studies focused primarily on short-term anatomic outcomes. Studies with longer term follow-up indicate that there 
are significant risks associated with $\mathrm{SC}$ and the potential need for reoperation continues indefinitely once mesh has been placed. The limitations of RCTs make it difficult to compare long-term morbidity of apical procedures, and it is likely that this important information will need to be provided by other approaches to POP research such as registries and practice databases. Current systematic reviews of apical surgery are based on only a handful of level 1 studies which could give a misleading interpretation of the overall efficacy and safety of the current options available for apical repair.

Financial disclaimer/Conflicts of interest None.

\section{References}

1. Benson JT, Lucente V, McClellan E (1996) Vaginal versus abdominal reconstructive surgery for the treatment of pelvic support defects: a prospective randomized study with long-term outcome evaluation. Am J Obstet Gynecol 175:1418-1422

2. Lo TS, Wang AC (1998) Abdominal colposacropexy and sacrospinous ligament suspension for severe uterovaginal prolapse: a comparison. J Gynecol Surg 14:59-64

3. Maher CF, Qatawneh AM, Dwyer PL, Carey MP, Cornish A, Schuleter PJ (2004) Abdominal sacrocolpopexy or vaginal sacrospinous colpopexy for vaginal vault prolapse: a prospective randomized study. Am J Obstet Gynecol 190:20-26

4. Maher C, Baessler K, Glazener CM, Adams EJ, Hagen S (2004) Surgical management of pelvic organ prolapse in women. Cochrane Database Syst Rev 4, CD004014

5. Maher C, Baessler K, Glazener CM, Adams EJ, Hagen S (2007) Surgical management of pelvic organ prolapse in women. Cochrane Database Syst Rev 3, CD004014

6. Maher C, Baessler K, Glazener CM, Adams EJ, Hagen S (2008) Surgical management of pelvic organ prolapse in women: a short version Cochrane review. Neurourol Urodyn 27(1):3-12

7. Maher C, Feiner B, Baessler K, Adams EJ, Hagen S, Glazener CM (2010) Surgical management of pelvic organ prolapse in women. Cochrane Database Syst Rev 4:CD004014

8. Maher CM, Feiner B, Baessler K, Glazener CM (2011) Surgical management of pelvic organ prolapse in women: the updated summary version Cochrane review. Int Urogynecol J 22(11):14451457

9. Maher C, Feiner B, Baessler K, Schmid C (2013) Surgical management of pelvic organ prolapse in women. Cochrane Database Syst Rev 4, CD004014

10. Barber MD, Maher C (2013) Apical prolapse. Int Urogynecol J 24(11):1815-1833

11. Siddiqui NY, Grimes CL, Casiano ER, Abed HT, Jeppson PC, Olivera CK, Sanses TV, Steinberg AC, South MM, Balk EM,
Sung VW, for the Society of Gynecologic Surgeons Systematic Review Group (2015) Mesh sacrocolpopexy compared with native tissue vaginal repair a systematic review and meta-analysis. Obstet Gynecol 125:44-55

12. Paraiso MF, Jelovsek JE, Frick A, Chen CC, Barber MD (2011) Laparoscopic compared with robotic sacrocolpopexy for vaginal prolapse: a randomized controlled trial. Obstet Gynecol 118: 1005-1013

13. Freeman RM, Pantazis K, Thomson A, Frappell J, Bombieri L, Moran P, Slack M, Scott P, Waterfield M (2013) A randomised controlled trial of abdominal versus laparoscopic sacrocolpopexy for the treatment of post-hysterectomy vaginal vault prolapse: LAS study. Int Urogynecol J 24:377-384

14. Maher CF, Feiner B, DeCuyper EM, Nichlos CJ, Hickey KV, O’Rourke PV (2011) Laparoscopic sacral colpopexy versus total vaginal mesh for vaginal vault prolapse: a randomized trial. Am J Obstet Gynecol 204:360.e1-360.e7

15. Rondini C, Braun HF, Alvarez J, Urzua M, Villegas R, Escobar M, Aros S, Wenzel C, Descouvieres C (2011) Prospective randomized study comparing high uterosacral vault suspension vs. abdominal sacral colpopexy for the correction of apical defects and vaginal vault prolapse. 36th IUGA Annual Meeting Abstract 088. Int Urogynecol J 22(Suppl 1):S87-S88

16. Silva WA, Pauls RN, Segal JL, Rooney CM, Kleeman SD, Karram MM (2006) Uterosacral ligament vault suspension: five-year outcomes. Obstet Gynecol 108:255-263

17. Miller D, Lucente V, Babin E, Beach P, Jones P, Robinson D (2011) Prospective clinical assessment of the transvaginal mesh technique for treatment of pelvic organ prolapse-5-year results. Female Pelvic Med Reconstr Surg 17:139-143

18. Gotthart PT, Aigmueller T, Lang PFJ, Ralph G, Bjelic-Radisic V, Tamussino K (2012) Reoperation for pelvic organ prolapse within 10 years of primary surgery for prolapse. Int Urogynecol J 23: $1221-1224$

19. Nygaard I, Brubaker L, Zyczynski HM, Cundiff G, Richter H, Gantz M, Fine P, Menefee S, Ridgeway B, Visco A, Warren LK, Zhang M, Meikle S (2013) Long-term outcomes following abdominal sacrocolpopexy for pelvic organ prolapse. JAMA 309(19): 2016-2024

20. Diwadkar GB, Barber MD, Feiner B, Maher C, Jelovsek JE (2009) Complication and reoperation rates after apical vaginal prolapse surgical repair: a systematic review. Obstet Gynecol $113: 367-372$

21. Jia X, Glazener C, Mowattt G, Jenkinson D, Fraser C, Bain C, Burr $J(2010)$ Systematic review of the efficacy and safety of using mesh in surgery for uterine or vaginal vault prolapse. Int Urogynecol J 21: $1413-1431$

22. Arsene E, Giraudet G, Lucot J, Rubod C, Cosson M (2015) Sacral colpopexy: long-term mesh complications requiring reoperation(s). Int Urogynecol J 26:353-358

23. van Geelen JM, Dwyer PL (2013) Where to for pelvic organ prolapse treatment after the FDA pronouncements? A systematic review of the recent literature. Int Urogynecol J 24:707-718 